\title{
AN INFINITE INTEGRAL INVOLVING MEIJER G-FUNCTION
}

\author{
K. L. ARORA AND S. K. KULSHRESHTHA
}

Abstract. In this note an infinite integral involving Meijer $G$-function and confluent hypergeometric functions is evaluated. Some particular cases of this integral are also discussed. The method used is based on operational calculus.

Main result. The result obtained in this paper is

$$
\begin{aligned}
& \int_{0}^{\infty} t^{\nu-1}(t+\eta)^{-1} e^{\left(\beta+\beta^{\prime}\right) t / 2} M_{k, m}(\beta t) M_{k^{\prime}, m^{\prime}}\left(\beta^{\prime} t\right) \\
& \cdot G_{p q}^{h l}\left(z t^{n} \mid \begin{array}{l}
a_{1}, \cdots, a_{p} \\
b_{1}, \cdots, b_{q}
\end{array}\right) d t \\
& =(2 \pi)^{1-n} \eta^{\nu-1} e^{-\left(\beta+\beta^{\prime}\right) \eta / 2} M_{-k, m}(\beta \eta) M_{-k^{\prime}, m^{\prime}}\left(\beta^{\prime} \eta\right) \\
& \cdot G_{p+n, q+n}^{h+n, l+n}\left(z \eta^{n} \mid \begin{array}{rr}
\Delta\left(n, n-\nu-m-m^{\prime}-1\right), a_{1}, \cdots, a_{p} \\
\Delta\left(n,-\nu-m-m^{\prime}\right),
\end{array}\right)
\end{aligned}
$$

where $n$ is a positive integer. The result holds for

$$
R\left(\nu+m+m^{\prime}+n b_{j}+2 n+1\right)>0, \quad j=1,2, \cdots, h .
$$

and if the following conditions are satisfied:

(i) $2(h+l+n) \geqq(p+q)$,

(ii) $|\arg z| \leqq(h+l-p / 2-q / 2+n) \pi$,

(iii) $R\left[\sum_{j=1}^{l} b_{j}-\sum_{j=1}^{h} a_{j}+p / 2-q / 2\right]<-1$.

The symbol $\Delta(s, a)$ represents $a / s,(a+1) / s, \cdots,(a+s-1) / s$.

In order to obtain result (1), we shall require the following result $[3$, p. 56] after making slight adjustments in the parameters

$$
\begin{aligned}
\int_{0}^{\infty} t^{\nu-1}(t+\eta)^{-1} e^{t\left(\beta+\beta^{\prime}\right) / 2} & M_{k, m}(\beta t) M_{k^{\prime}, m^{\prime}}\left(\beta^{\prime} t\right) d t \\
= & \eta^{\nu-1} e^{-\eta\left(\beta+\beta^{\prime}\right) / 2} M_{-k, m}(\eta \beta) M_{-k^{\prime}, m^{\prime}}\left(\eta \beta^{\prime}\right) \\
& \cdot \Gamma\left(\nu+m+m^{\prime}+1\right) \Gamma\left(-\nu-m-m^{\prime}\right) .
\end{aligned}
$$

the formula $[1$, p. 4$]$

Received by the editors January 16, 1970.

A MS 1968 subject classifications. Primary 3035, 3321, 4440; Secondary 3315, 3320, 3345.

Key words and phrases. Operational calculus, infinite integral, confluent hypergeometric functions, special functions, absolute convergence. 


$$
\prod_{r=0}^{m-1} \Gamma\left(z+\frac{r}{m}\right)=(2 \pi)^{m / 2-1 / 2} m^{1 / 2-m z} \Gamma(m z)
$$

and the following representation of Meijer $G$-function [2] as contour integral

$$
\begin{aligned}
G_{p q}^{h l}\left(x \mid \begin{array}{l}
a_{1}, \cdots, a_{p} \\
b_{1}, \cdots, b_{q}
\end{array}\right) & \\
& =\frac{1}{2 \pi i} \int_{L} \frac{\prod_{j=1}^{h} \Gamma\left(b_{j}-s\right) \prod_{j=1}^{l} \Gamma\left(1-a_{j}+s\right)}{\prod_{j=h+1}^{q} \Gamma\left(1-b_{j}+s\right) \prod_{j=l+1}^{p} \Gamma\left(a_{j}-s\right)} x^{s} d s .
\end{aligned}
$$

Proof. On substituting for

$$
G_{p q}^{h l}\left(x \mid \begin{array}{l}
a_{1}, \cdots, a_{p} \\
b_{1}, \cdots, b_{q}
\end{array}\right)
$$

from (4) in the integrand (1), changing the order of integration and evaluating the integral with the help of (2), one gets

$\eta^{\gamma-1} e^{-\eta\left(\beta+\beta^{\prime}\right) / 2} M_{-k, m}(\eta \beta) M_{-k^{\prime}, m^{\prime}}\left(\eta \beta^{\prime}\right)$

(5)

$$
\frac{1}{2 \pi i} \int_{L} \frac{\prod_{j=1}^{h} \Gamma\left(b_{j}-s\right) \prod_{j=1}^{l} \Gamma\left(1-a_{j}+s\right) \Gamma\left(\nu+m+m^{\prime}+1+n s\right) \Gamma\left(-\nu-m-m^{\prime}-n s\right)}{\prod_{j=h+1}^{q} \Gamma\left(1-b_{j}+s\right) \prod_{j=l+1}^{p} \Gamma\left(a_{j}-s\right)}\left(z \alpha^{n}\right)^{s} d s .
$$

If we now apply (3) and interpret with the help of (4), we obtain the main result (1).

The contour taken here is a straight line along the imaginary axis extending from $-i \infty$ to $+i \infty$ with indentations if necessary so that all the poles of $\Gamma\left(b_{j}-s\right), j=1,2, \cdots, h, \Gamma\left(-\nu-m-m^{\prime}-n s\right)$ are to the right of it and the poles of $\Gamma\left(1-a_{k}+s\right), k=1,2, \cdots, l$, and $\Gamma\left(\nu+m+m^{\prime}+1-n b_{j}\right), j=1,2, \cdots, h$, are to the left of it. This is possible if $R\left(a_{t}-1\right)<0$, and $R\left(\nu+m+m^{\prime}+1-n b_{j}\right)>0$, $j=1,2, \cdots, h ; t=1,2, \cdots, l$. The change of the order of integration in the proof is also justified. Let us first investigate the convergence of integral (5). We put $s=i t$ and take the limit as $t \rightarrow \infty$. Since $|\Gamma(x+i t)|=\sqrt{ }(2 \pi)|t|^{x-1 / 2} e^{-\alpha|t| / 2}$ the absolute value of the above integral is comparable with

$$
\exp \left\{-\frac{\pi}{2}(2 h+2 l-p-q+2 n)|t|\right\} e^{\phi t}|t|^{\sum_{j=1}^{q} b_{j}-\sum_{j=1}^{p} a_{j}+p / 2-q / 2}
$$

where $z=R e^{i \phi}$. Hence if $(2 \alpha+2 \beta)>\gamma+\delta$, then the integral is convergent if either $\phi<(h+l-p / 2-q / 2) \pi$ or $\phi=h+l-p / 2-q / 2$, then 


$$
R\left[\sum_{j=1}^{q} b_{j}-\sum_{j=1}^{p} a_{j}+p / 2-q / 2\right]<-1
$$

while if $(2 h+2 l+2 n)=p+q$, then the integral is convergent only if

$$
\phi=0=(h+l-p / 2-q / 2+n) \pi
$$

and

$$
R\left[\sum_{j=1}^{q} b_{j}-\sum_{j=1}^{p} a_{j}+\frac{p}{2}-\frac{q}{2}\right]<-1 .
$$

Thus we see that the change of the order of integration is permissible under the conditions given with (1). As with them the integral (4) is absolutely convergent. The inner integral after change of the order of integration is absolutely convergent and the resulting integral (5) is also convergent.

Since $G$-function is a generalization of a great many special functions appearing in applied mathematics, many particular cases can be obtained from (1) by giving particular values to the parameters. We give below a few of the particular cases.

(i) Taking $h=q=4, l=0, p=2, a_{1}=a, a_{2}=a+\frac{1}{2}, b_{1}=a+b, b_{2}=a+c$, $b_{3}=a-c$ and $b_{4}=a-b$ and using the following result $[1$, p. 219]

$$
G_{24}^{40}\left(\begin{array}{l}
x \mid \begin{array}{l}
a, a+\frac{1}{2} \\
a+b, a+c, a-c, a-b
\end{array}
\end{array}\right)=2 \pi^{-1 / 2} x^{a} K_{b+c}(\sqrt{ } x) K_{b-c}(\sqrt{ } x),
$$

we obtain

$$
\begin{aligned}
& \int_{0}^{\infty} t^{\nu+n a-1}(t+\eta)^{-1} e^{\left(\beta+\beta^{\prime}\right) t / 2} M_{k, m}(\beta t) M_{k^{\prime}, m^{\prime}}\left(\beta^{\prime} t\right) \\
& \cdot K_{b+c}\left(\sqrt{ }\left(z t^{n}\right)\right) K_{b-c}\left(\sqrt{ }\left(z t^{n}\right)\right) d t \\
& =\frac{\pi^{3 / 2-n} \eta^{\nu-1}}{2^{n} z^{a}} e^{-\left(\beta+\beta^{\prime}\right) \eta / 2} M_{-k, m}(\beta \eta) M_{-k^{\prime}, m^{\prime}}\left(\beta^{\prime} \eta\right) \\
& \cdot G_{n+2, n+4}^{n+4, n}\left(z \eta^{n} \mid \begin{array}{l}
\Delta\left(n, n-\nu-m-m^{\prime}-1\right), a, a+\frac{1}{2} \\
\Delta\left(n,-\nu-m-m^{\prime}\right), a+b, a+c, a-c, a-b
\end{array}\right),
\end{aligned}
$$

where

$$
R\left\{\nu+m+m^{\prime}+2 n+1+n(a+b, a+c, a-c, a-b)\right\}>0,
$$

$n$ being a positive integer and $|\arg z| \leqq(n+1) \pi$.

(ii) Taking $h=q=4, l=0, p=2, a_{1}=\frac{1}{2}+a, a_{2}=\frac{1}{2}-a, b_{1}=0, b_{2}=\frac{1}{2}$, $b_{3}=b$ and $b_{4}=-b$ and using the following result $[1$, p. 218] 


$$
G_{24}^{40}\left(x \mid \begin{array}{c}
\frac{1}{2}+a, \frac{1}{2}-a \\
0, \frac{1}{2}, b,-b
\end{array}\right)=\sqrt{ } \pi x^{-1 / 2} W_{a, b}(2 \sqrt{ } x) W_{-a, b}(2 \sqrt{ } x)
$$

we obtain

$$
\int_{0}^{\infty} t^{\nu-n / 2-1}(t+\eta)^{-1} e^{\left(\beta+\beta^{\prime}\right) t / 2} M_{k, m}(\beta t) M_{k^{\prime} m^{\prime}}\left(\beta^{\prime} t\right)
$$

$$
\text { - } W_{a, b}\left(2 \sqrt{ }\left(z t^{n}\right)\right) W_{-a, b}\left(2 \sqrt{ }\left(z t^{n}\right)\right) d t
$$

$$
\begin{aligned}
& =2^{1-n} \pi^{1 / 2-n} \eta^{\nu-1} z^{1 / 2} e^{-\eta\left(\beta+\beta^{\prime}\right) / 2} M_{-k, m}(\eta \beta) M_{-k^{\prime}, m^{\prime}}\left(\eta \beta^{\prime}\right) \\
& \cdot G_{n+2, n+4}^{n+4, n}\left(z \eta^{n} \begin{array}{l}
\Delta\left(n, n-\nu-m-m^{\prime}+1\right), \frac{1}{2}+a, \frac{1}{2}-a \\
\Delta\left(n,-\nu-m-m^{\prime}\right), 0, \frac{1}{2}, b,-b
\end{array}\right)
\end{aligned}
$$

where $R\left[\nu+m+m^{\prime}+2 n+1+n\left(0, \frac{1}{2}, b,-b\right)\right]>0, n$ being a positive integer and $|\arg z| \leqq(n+1) \pi$.

(iii) Let $h=1, l=p=2, q=4, a_{1}=\frac{1}{2}+a, a_{2}=a, b_{1}=a+b, b_{2}=a-c$, $b_{3}=a+c, b_{4}=a-b$. Using the result $[1$, p. 218]

$$
G_{24}^{12}\left(x \mid \begin{array}{l}
a+\frac{1}{2}, a \\
b+a, a-c, a+c, a-b
\end{array}\right)=\sqrt{ } \pi x^{a} J_{b+c}(\sqrt{ } x) J_{b-c}(\sqrt{ } x)
$$

we get

$$
\begin{aligned}
& \int_{0}^{\infty} t^{\nu+n a-1}(t+\eta)^{-1} e^{t\left(\beta+\beta^{\prime}\right) / 2} M_{k, m}(\beta t) \\
& \cdot M_{k^{\prime}, m^{\prime}}\left(\beta^{\prime} t\right) J_{b+c}\left(\sqrt{ }\left(z t^{n}\right)\right) J_{b-c}\left(\sqrt{ }\left(z t^{n}\right)\right) d t \\
& =\frac{\pi^{1 / 2-n} \eta^{\nu-1}}{2^{n} z^{a}} e^{-\eta\left(\beta+\beta^{\prime}\right) / 2} M_{-k, m}(\eta \beta) M_{-k^{\prime}, m^{\prime}}\left(\eta \beta^{\prime}\right) \\
& \cdot G_{n+2, n+4}^{n+1, n+2}\left(z \eta^{n} \mid \begin{array}{l}
\Delta\left(n, n-\nu-m-m^{\prime}-1\right), a+\frac{1}{2}, a \\
\Delta\left(n,-\nu-m-m^{\prime}\right), b+a, a-c, a+c, a-b
\end{array}\right),
\end{aligned}
$$

where $R\left[\nu+m+m^{\prime}+2 n+1+n(b+a)\right]>0, n$ being a positive integer and $|\arg z| \leqq n \pi$.

(iv) Let $h=l=p=2, q=4, a_{1}=a, a_{2}=a+\frac{1}{2}, b_{1}=b, b_{2}=c, b_{3}=2 a-c$, $b_{4}=2 a-b$. Using the following result $[1$, p. 218]

$$
G_{24}^{22}\left(x \mid \begin{array}{l}
a, a+\frac{1}{2} \\
b, c, 2 a-c, 2 a-b
\end{array}\right)=2 \sqrt{ } \pi \cdot x^{a} I_{b+c-2 a}(\sqrt{ } x) K_{b-c}(\sqrt{ } x)
$$

we obtain 


$$
\begin{aligned}
& \int_{0}^{\infty} t^{\nu+n a-1}(t+\eta)^{-1} e^{t\left(\beta+\beta^{\prime}\right) / 2} M_{k, m}(\beta t) \\
& \cdot M_{k^{\prime}, m^{\prime}}\left(\beta^{\prime} t\right) I_{b+c-2 a}\left(\sqrt{ }\left(z t^{n}\right)\right) K_{b-c}\left(\sqrt{ }\left(z t^{n}\right)\right) d t \\
& =\frac{\pi^{1 / 2-n} \eta^{\nu-1}}{2^{n} z^{a}} e^{-\eta\left(\beta+\beta^{\prime}\right) / 2} M_{-k, m}(\beta \eta) M_{-k^{\prime}, m^{\prime}}\left(\eta \beta^{\prime}\right) \\
& \cdot G_{n+2, n+4}^{n+2, n+2}\left(z \eta^{n} \mid \begin{array}{l}
\Delta\left(n, n-\nu-m-m^{\prime}-1\right), a, a+\frac{1}{2} \\
\Delta\left(n,-\nu-m-m^{\prime}\right), b, c, 2 a-c, 2 a-b
\end{array}\right)
\end{aligned}
$$

where $R\left[\nu+m+m^{\prime}+2 n+1+n(b, c)\right]>0, n$ being a positive integer and $|\arg z| \leqq(n+1) \pi$.

\section{REFERENCES}

1. A. Erdélyi et al., Higher transcendental functions. Vol. I, McGraw-Hill, New York, 1953. MR 15, 419.

2. C. S. Meijer, On G-function 1, Nederl. Akad. Wetensch Proc. 49 (1946), 227-237 = Indag. Math. 8 (1946), 124-134. MR 8, 156.

3. L. J. Slater, Confluent hypergeometric functions, Cambridge Univ. Press, New York, 1960. MR 21 \#5753.

Punjab Engineering College, Chandigarh, India 